\title{
Investigating the relationship between intergroup physical contact and attitudes towards foreigners: the mediating role of quality of intergroup contact
}

\author{
Soraya E Shamloo ${ }^{\text {Corresp., }}{ }^{1}$, Andrea Carnaghi ${ }^{1}$, Carlo Fantoni ${ }^{1}$ \\ 1 Department of Life Sciences, University of Trieste, Trieste, Italy \\ Corresponding Author: Soraya E Shamloo \\ Email address: sorayaelizabeth.shamloo@phd.units.it
}

Recent research has shown that a brief, casual touch administered by an outgroup member reduces prejudice towards the group to which the toucher belongs. In this study, we take the research on physical contact and prejudice a step further by addressing the relation between individuals' amount of Experienced Intergroup Physical Contact (EIPC), across distinct contexts and involving different body parts, and attitudes towards foreign people. Specifically, we hypothesized that the amount of EIPC would be positively associated with both quantity and quality of intergroup contact, but that only quality would mediate the relationship between the amount of EIPC and outgroup attitudes, being quality more directly linked to the evaluative component of outgroup attitudes. To attain this aim, we asked participants to self-report the amount of EIPC, the quantity and quality of their intergroup contact and their attitudes towards foreign people. Consistent with our hypothesis: (1) As EIPC increased, positive attitudes towards foreign people increased; (2) Higher levels of EIPC were associated with better quality and higher quantity of intergroup contact; (3) Only quality of intergroup contact mediated the relationship between the amount of EIPC and attitudes towards foreign people. Results were discussed in relation to research on intergroup contact and physical contact. 
1 Investigating the relationship between intergroup physical contact and attitudes towards

2 foreigners: the mediating role of quality of intergroup contact

3

4 Soraya Elizabeth Shamloo ${ }^{1 *}$, Andrea Carnaghi $^{1 *}$, Carlo Fantoni $^{1}$

$5 *$ These authors contributed equally to this work

6

$7 \quad{ }^{1}$ Department of Life Sciences, University of Trieste, Via Weiss 21, 34100, Trieste, Italy

8

9 Corresponding Author:

10 Soraya Shamloo ${ }^{1}$

11

12

Email address: sorayaelizabeth.shamloo@phd.units.it 
14

15

16

17

18

19

20

21

22

23

24

25

26

27

28

29

\begin{abstract}
Recent research has shown that a brief, casual touch administered by an outgroup member reduces prejudice towards the group to which the toucher belongs. In this study, we take the research on physical contact and prejudice a step further by addressing the relation between individuals' amount of Experienced Intergroup Physical Contact (EIPC), across distinct contexts and involving different body parts, and attitudes towards foreign people. Specifically, we hypothesized that the amount of EIPC would be positively associated with both quantity and quality of intergroup contact, but that only quality would mediate the relationship between the amount of EIPC and outgroup attitudes, being quality more directly linked to the evaluative component of outgroup attitudes. To attain this aim, we asked participants to self-report the amount of EIPC, the quantity and quality of their intergroup contact and their attitudes towards foreign people. Consistent with our hypothesis: (1) As EIPC increased, positive attitudes towards foreign people increased; (2) Higher levels of EIPC were associated with better quality and higher quantity of intergroup contact; (3) Only quality of intergroup contact mediated the relationship between the amount of EIPC and attitudes towards foreign people. Results were discussed in relation to research on intergroup contact and physical contact.
\end{abstract}




\section{Introduction}

32 Challenges raised by conflicting intergroup relations have always been of primary interest for social sciences. Given the growing anti-immigration public opinion (e.g., Blinder \& Allen, 2016), research focused on finding ways to promote harmonious intergroup relations and contrast social exclusion is highly needed.

Accumulated evidence has addressed the role played by intergroup contact in prejudice revision. In this respect, a wide range of studies has documented the effectiveness of direct, positive interactions with outgroup members in improving attitudes towards the outgroup (Allport, 1954; Pettigrew \& Tropp, 2006). Not only direct contact but also indirect forms of contact, which do not require people to actually interact with each other, have shown to be effective in reducing prejudice towards different groups (Lemmer \& Wagner, 2015; Brown \& Paterson, 2016). Likewise direct contact, indirect contact bases its effectiveness on the possibility of experiencing social interactions, may this be in the form of mental simulation, as in the case of imagined contact (e.g., Crisp \& Turner, 2009), or in the form of media portrayals in which one is exposed to positive interactions between groups, as in the case of vicarious contact (e.g., Mazziotta, Mummendey, \& Wright, 2011).

In everyday life, people connect with others by relying on verbal but also on nonverbal communication, as in the case of physical contact. Touch in particular plays a pivotal role in human communication. Indeed, touch is at the basis of early human communicative interactions (Field, 2001; Hertenstein et al., 2006) and develops into an elaborated symbolic system (Jones \& Yarbrough, 1985). Touch can convey different emotions (Hertenstein et al., 2006) and thus plays a major role in shaping interpersonal interactions (Gallace \& Spence, 2010 for a review). As far as physiological responses to touch are concerned, studies have shown that touch increases levels 
54 of oxytocin thus demonstrating its comforting and positive effects on well-being (e.g., Holt-

55 Lunstad, Birmingham, \& Light, 2008). At the interpersonal level, the use of physical contact

56 during interactions also promotes positive evaluations towards the toucher (Erceau \& Guéguen,

57 2007), enhances cooperation within groups (Kraus, Huang \& Keltner, 2010) and elicits pro-

58

59

60

social behaviour (e.g., Guéguen \& Fischer-Lokou, 2003). Within the literature on embodied cognition, empirical efforts were carried out to understand how aspects of social relationships are regulated by embodied cues, such as touch (Gallese, 2001; Decety \& Grèzes, 2006; Smith, 2008; Fantoni et al., 2016). Based on this rationale, Fiske (2004) suggested that physical contact/touch among members is highly common within a specific form of relationship, namely the Communal Sharing Relationships. Communal Sharing Relationships are typical of close relationships in which group members share resources and focus on members' communality. These forms of relationships are said to be embodied by sharing among other things the use of physical proximity, touch and synchronized body movements (Smith, 2008) which may enhance cohesiveness among individuals (e.g., Wiltermuth et al., 2009).

Recently the impact of physical contact in intergroup relations has been partially addressed by research relying on the imagined intergroup contact paradigm (Shamloo et al., in press). In general, this paradigm requires that participants mentally simulate an intergroup encounter (Crisp et al., 2010). For instance, participants who were requested to imagine interacting with an outgroup member, reported lower levels of prejudice towards the outgroup as a whole than participants in a control condition (Turner, Crisp \& Lambert, 2007). Research that relies on fMRi (i.e., functional magnetic resonance imaging) and PET (i.e., positron emission tomography) shows that mental imagery involves, at least in part, similar brain networks as those recruited in actual perception and emotion (Kosslyn et al., 2001). Based on this evidence, Turner 
77 and colleagues (2007) argue that the mental simulation of intergroup encounters allows the

78 recruitment of mental structures that are present also during actual encounters with outgroup

79 members (Crisp et al., 2009). In line with this insight, Hodson and colleagues (2015) required

80 participants to imagine a cooperative interaction with a homeless person, in which physical

81 contact was encouraged, or to simply imagine meeting a homeless person. Also, a neutral

82 imagined scenario was included in the experimental design as a control condition. Results

83 indicated that, compared to the other conditions, only participants who imagined a cooperative

84 interaction that involved a physical contact with a homeless person reported a weaker association

85 between outgroup disgust and outgroup trust, which in turn mediated the relationship between

86 disgust sensitivity and outgroup prejudice. Similarly, Choma and colleagues (2014) first assessed

87 participants' prejudice, and then participants were asked to imagine physically interacting with

88 an outgroup member. In this case the imagined physical encounter was based on team-building

89 exercises (i.e., "thumb war" session), which required cooperation with the outgroup member.

90 Before and after engaging in actual physical contact (i.e., wrist loops) with the outgroup member,

91 participants' prejudice was assessed again. Results showed that following the imagined physical

92 contact task, participants' levels of prejudice significantly decreased and remained stable across

93 the following sessions.

94 The mentioned studies (Choma et al., 2014 and Hodson et al., 2015) suggest that physical

95 contact may ameliorate outgroup attitudes. Nevertheless, these studies involved cooperation

96 (e.g., trust-building or team-building exercises) between individuals which may have played a

97 role in this respect (Gaertner et al., 1990). Said otherwise, as in this research the (imagined)

98 physical encounter with an outgroup member was associated with a cooperative setting, and

99 given that intergroup cooperation improves per se outgroup attitudes (Gaertner et al., 1990), the 
100 unique contribution of intergroup physical contact in ameliorating intergroup relations was only

101 partially addressed by the above-mentioned research. To our knowledge, only a research carried

102 out by Seger and colleagues (2014) has restricted its analyses to the impact of actual intergroup

103 physical contact per se on outgroup attitudes. Specifically, Seger and colleagues (2014) tested

104 the idea that a real touch, which is at the basis of Communal Sharing Relationships, would not

105 only positively affect the evaluation of the individual toucher but also the group to which the 106 toucher belongs.

107 In this respect, Seger and colleagues (2014) showed that a brief and casual touch (i.e., a

108 single physical contact encounter) on the shoulder of European-American participants,

109 performed by an African-American experimenter, reduced prejudice towards African-Americans,

110 compared to when participants did not receive any touch. In other words, this research

111 demonstrated that intergroup touch positively impacts on the evaluation of the outgroup toucher,

112 and this positive experience generalizes to the toucher's group as a whole.

113 This research (Seger et al. 2014) underlines the potential role played by intergroup touch

114 in reducing outgroup prejudice and opens up the question of why intergroup physical contact

115 (i.e., physical contact between ingroup and outgroup members) contributes to the improvement

116 of outgroup attitudes. Notwithstanding the importance of this research, the psychological

117 mechanism that brings perceivers, who have experienced a physical encounter with an outgroup

118 member, to improve their attitudes towards the outgroup as a whole has not been addressed yet.

119 Said otherwise, the 'how' physical contact in intergroup relations shapes intergroup attitudes has

120 not been investigated by previous research (Choma et al., 2014; Seger et al., 2014; Hodson et al.,

121 2015). The current research intends to fill this gap by analyzing the role of potential mediating

122 variables in the relation between intergroup physical contact and outgroup attitudes. In other 
123 words, we first intend to gather further evidence on the positive association between intergroup

124 physical contact and outgroup attitudes thus strengthening the idea that enhanced levels of

125 intergroup physical experience are associated with more positive attitudes towards the outgroup,

126 and, more importantly, analyze the potential mediators that could account for the relation in

127 question. To reach this aim, we analyze the relationship between individuals' amount of

128 Experienced Intergroup Physical Contact (i.e., EIPC) with foreigners and attitudes towards this

129 outgroup. Differently from Seger and colleagues' operationalization of intergroup physical

130 contact, which involved a single casual touch, we assess participants' amount of EIPC, including

131 a variety of types of physical contact involving different body parts and across a variety of

132 contexts, thus relying on a comprehensive experience of physical contact with foreign

133 individuals (i.e., outgroup). We hypothesize that the extent to which participants have

134 experienced different intergroup physical contact encounters with outgroup members would

135 work as the basis for the generalization of these positive experiences to the outgroup as a whole

136 (Hypothesis 1). Second, we reasoned that intergroup physical encounters may facilitate

137 intergroup contact which ultimately improves outgroup attitudes. As a case in point, recent

138 research has shown that physical contact helps ameliorating close interpersonal relations

139 (Gulledge, Gulledge \& Stahmannn, 2003), backs pro-social behavior (Guéguen \& Fischer-

140 Lokou, 2003) and enhances cooperation (Kraus, Huang \& Keltner, 2010). Also, physical contact

141 is at the basis of cooperative relationships, in which individuals share a close interpersonal tie

142 (Fiske, 2004) and create a sense of communality with others (Seger et al., 2014). If physical

143 contact itself has the power of triggering such outcomes at the interpersonal level, it may be

144 reasonable to think that it may exert similar effects at the intergroup level. Said otherwise,

145 physical contact could enhance the perceived cooperation, pleasantness and the depth of 
146 intergroup encounters. These characteristics of an intergroup interaction are typically

147 operationalized by the quality of intergroup contact. Hence, we would expect that higher levels

148 of physical contact would predict better overall quality of intergroup contact. Not only physical

149 contact might improve the quality of intergroup contact but it might also enhance the quantity of

150 intergroup encounters. Indeed, it is plausible that the positive experience triggered by engaging

151 in intergroup situations, in which also physical contact is involved, may enhance the opportunity

152 of intergroup encounters by weakening the common avoidance-like reactions towards outgroup

153 members (e.g., Paladino \& Castelli, 2008; Bianchi, Carnaghi \& Shamloo, 2018) and by

154 encouraging more approach-like behaviors towards outgroup members, which ultimately impact

155 on the frequency of intergroup encounters (Kawakami et al., 2007).

156 We thus hypothesize (Hypothesis 2) that higher levels of EIPC will be associated with a

157 more positive appraisal of the intergroup contact (i.e., quality of intergroup contact) as well as

158 enhanced opportunities of intergroup encounters (i.e., quantity of intergroup contact).

159 The distinct impact of quantity and quality of intergroup contact on different aspects of

160 outgroup attitudes contributes to clarify the potential mediating role of these variables in the

161 relation between the amount of EIPC and outgroup attitudes. Indeed, keeping these two aspects

162 of intergroup contact distinct would help to better understand which aspect of intergroup contact

163 plays a major role in the relationship between intergroup physical contact and outgroup attitudes.

164 This decision is supported by empirical evidence attesting to a different and distinct association

165 between quality/quantity of intergroup contact, and outgroup attitudes and beliefs. Specifically,

166 research which has focused on these two aspects of intergroup contact separately, has pointed out

167 that quality, more so than quantity of intergroup contact, greatly predicts positive attitudes

168 toward the outgroup (Islam and Hewstone, 1993; Stephan, Diaz-Loving, \& Duran, 2000; Viki et 
169 al., 2006). By contrast, quantity of intergroup encounters greatly impacts on perceived outgroup

170 variability (Islam and Hewstone, 1993). Hence, quality, more so than quantity of intergroup

171 contact seems to be associated with affective, evaluative-based reactions towards the outgroup

172 (e.g., prejudice), while the frequency of intergroup contact is likely to be associated with the

173 cognitive representation of the outgroup (e.g., outgroup homogeneity). For this reason, it might

174 be plausible that the quality, rather than the quantity of intergroup contact would more likely

175 predict participants' prejudice towards the outgroup (Hypothesis 3 ). In the current study we

176 assessed participants' evaluative-based reactions towards the outgroup by means of the General

177 Evaluation Scale (Wright et al., 1997), which taps participants' evaluative responses to the target 178 outgroup.

179 In sum, given that physical contact would positively predict the positive appraisal of 180 intergroup encounters (quality of intergroup contact) as well as the frequency of intergroup 181 encounters (quantity of intergroup contact), and due to the preferential association of quality over 182 quantity of intergroup contact with prejudice, we hypothesized that quality of intergroup contact 183 would be a solid candidate to mediate the association between the amount of EIPC and attitudes 184 towards the outgroup. Therefore, we put forward that increased amounts of EIPC will be 185 associated with more positive attitudes towards the outgroup because EIPC positively shapes the 186 quality of the intergroup contact (Hypothesis 4).

187

188 Materials \& Methods

189 Pilot study

190 Prior to the main research, a scale tapping participants' amount of experienced physical contact

191 with known people (i.e., EPC-known person scale, see Appendix) was developed and taken into 
192 examination, thus allowing us to use the scale and adapt it to the purpose of the main study. A

193 principle component analysis was performed in order to analyze its factor structure. In addition,

194 we tested its reliability, and then its association with a proxy of physical contact, namely the

195 Comfortable Interpersonal Distance scale (i.e., CID scale; Duke \& Nowicki, 1972) in order to 196 test convergent and discriminant validity.

197

198

199

200

201

\section{Results}

\section{Measures}

\section{Participants and Procedure}

Ninety-four participants ( $n=74$ female and $n=19$ male participants, $n=1$ did not report this information; age: $M=20.53, S D=3.99$ ) took part in the pilot study. Participants were told we were interested in collecting opinions regarding the social domain. Participants were provided a questionnaire and answered the following measures.

\section{Amount of experienced physical contact with a known person (EPC-known person).}

A twelve-item scale tapping participants' amount of experienced physical contact with known people involving different body parts and regarding different situations was administered to participants. Participants rated the amount of experienced physical contact with a known person on a 5-point scale, ranging from 1 (= never $)$ to 5 (= often $)^{1}$.

Comfort with interpersonal distance (CID). Comfort with interpersonal distance was measured by using the Comfortable Interpersonal Distance scale (i.e., CID scale; Duke \& Nowicki, 1972) with a known person (i.e., CID-known person) and a stranger (i.e., CIDstranger). Higher scores in this scale indicated lower comfort with interpersonal distance. 
ratings of the items pertaining to the amount of experienced physical contact regarding known

217 people. Results revealed a single factor structure that explained $47.7 \%$ of variance and factor

218 loading ranged between .45 and .83 (see Table 1). Alpha could not be increased by eliminating

219 any item. The reliability of the scale was high $(\alpha=.89)$. Therefore, participants' ratings on the

220 EPC-known person were averaged to reach a single composite measure of experienced physical $221 \operatorname{contact}(M=3.93, S D=.70)$.

222 We then proceeded by testing the association of the EPC-known person scale with the

223 CID-known person $(M=1.11, S D=.64)$ and CID-stranger scale $(M=2.71, S D=1.03)$. As the

224 CID scale in general is a measure of how comfortable people feel with interpersonal distance, it 225 might represent a proxy of physical contact. Hence, participants' ratings on the EPC-known 226 person scale were regressed on their reactions to the CID-known person and CID-stranger scale.

227 The CID-known person negatively predicted the EPC-known person $(\beta=-.27, t=2.12, p=.04)$,

228 indicating that the more comfortable one felt with interpersonal distance with a known person, 229 the higher the amount of physical contact experienced with known people. On the other hand, the 230 CID-stranger positively predicted the EPC-known person $(\beta=.28, t=2.15, p=.04)$, showing 231 that the less comfortable one felt with interpersonal distance with a stranger the higher the 232 amount of physical contact experienced with known people.

233 To sum up, the EPC-known person scale has a good reliability and shows significant 234 association with a proxy of physical contact and thus will be used in the main study. 
237 Although past research (Choma et al., 2014; Seger et al., 2014; Hodson et al., 2015) has focused

238 on an experimental approach to study the effects of intergroup physical contact on attitudes

239 towards the outgroup, in the current study we tackle this issue by using a correlational approach

240 for two distinct, albeit related reasons. Firstly, this method has been largely used in research

241 addressing the relationship between intergroup contact and outgroup attitudes, and constitutes a

242 reliable approach to the study of social psychological processes involved in the intergroup

243 contact (e.g., Voci \& Hewstone, 2003; Turner, Hewstone \& Voci 2007; Christ et al., 2010).

244 Secondly, the current work aims to study the frequency of different types of physical contact

245 involving different body parts and across a variety of contexts. In other words, we intend to

246 capture a broad and comprehensive experience of physical contact with foreign individuals (i.e.,

247 outgroup), rather than a single intergroup touch (Seger et al., 2014), across different everyday

248 life contexts, rather than in a specific context (Choma et al., 2014, Hodson et al., 2015). For this

249 reason, a survey would match the current aim as it does not constrain the analyses to a limited

250 number and types of physical experiences as well as contexts in which the intergroup physical

251 experiences occurred. Although relying on a cross-sectional design allows us to explore how

252 intergroup physical contact relates to outgroup attitudes in a natural setting, thus enhancing the

253 ecological validity of our findings, it prevents us from strong claims about the causal relationship

254 among variables.

\section{Participants and Procedure}

257 We decided to rely on a sample of 100 participants. This decision was backed by a sensitivity analyses (G Power 3.1; Faul et al., 2007), $\alpha$ err. prob. $=.05$, Power $(1-\beta$ err. prob. $)=$

$259.8, N=100$, which indicated a Minimal Detectable Effect (MDE) size $\mathrm{f}=.11$. Hence, the 
260 smallest real effect size which we would be able to detect (at $80 \%$ power) with this sample size

261 falls within the small-effect size area (Cohen, 1988). We decided to collect more than 100

262 participants to be sure to reach the estimated sample size given the probability of coming across

263 missing data. One hundred eleven students from a university in northern Italy participated in this

264 study. Two participants did not report their gender and could not be entered in the lme model

265 which treated this variable as a covariate. The remaining participants did not fully rate the

266 variables included in the mediation models ( $n=1$ on the EIPC scale; $n=2$ on quality of

267 intergroup contact; $n=1$ on the prejudice scale; $n=1$ on both the prejudice scale and quality of

268 intergroup contact). Given that we relied on aggregated measures of participants' ratings as

269 indexing the variable under examination, the exclusion of the participants who did not rate all the

270 items of a given scale is needed as calculating the synthetic value of the scale on a different and

271 limited number of rated items would undermine the reliability of the entire measures

272 (Supplemental Analyses S1). The final sample comprised $n=58$ female participants and $n=46$

273 male participants (age: $M=22.12, S D=2.99$ ). Among these participants, $n=98$ were Italians, $n$

$274=5$ were not Italians and $n=1$ indicated two nationalities. The current sample size approximated

275 the $\mathrm{N}$ rule.

276 Prior to filling in the questionnaire, the researcher provided participants with the written

277 consent and assured they had understood it.

278 We decided to rely on the outgroup target 'foreigners' for different reasons. First, Asbrock

279 and colleagues (2014) found that when participants are asked to think about foreigners, they tend

280 to indicate and think about the most salient ethnic minorities in a given country (p. 6, Asbrock et

281 al., 2014), thus suggesting a large overlap between the term 'foreigners' and ethnic minorities. In

282 line with the above-mentioned claims, several studies rooted in the intergroup relation tradition 
283 have often measured prejudice towards foreigners in general (e.g., Liebkind \& McAlister, 1999;

284 Raijman, Semyonov, \& Schmidt, 2003; Christ et al., 2010). Second, the term 'foreigners', at

285 least in the Italian context, is typically used as synonym of nonItalian, ethnic minorities and

286 recently employed by the National Institute of Statistics to assess Italian respondents' attitudes

287 towards ethnic minorities (ISTAT, 2018).

288 Quantity and quality of intergroup contact, the amount of EIPC with foreign people and 289 outgroup attitudes were self-assessed. Half of the participants rated the measures in the above-

290 mentioned order, whereas the other half rated outgroup attitudes first, then quantity and quality

291 of intergroup contact and then the amount of EIPC with foreign people (i.e., order of

292 presentation).

293

294 Measures

295

Quantity and quality of contact with foreign people. Two items (adapted from Voci \& 296

Hewstone, 2003) tapped the quantity of intergroup contact $(\alpha=.71)$, namely: "How many

297

foreign people do you know?" (None-More than 10), "How frequently do you have contact with

298

foreign people?" (Never-Very frequently). All answers were given on a 5-point scale. As for the

299

quality of intergroup contact ( $\alpha=.75$; adapted from Voci \& Hewstone, 2003), participants were

300

asked: "When you meet foreign people, in general you find the contact..." and presented with

301

three adjectives: pleasant (piacevole in Italian), cooperative (cooperativo in Italian), superficial

302 (superficial in Italian). Answers ranged from 1 (= Not at all) to 5 (=Totally). As an aggregated

303

measure of the quantity of intergroup contact and quality of intergroup contact, we relied on the

304 median value of the corresponding items. 
structure yielded by the EPC- known person scale used in the pilot study, its good reliability and association with a proxy of physical contact (i.e., the comfortable interpersonal distance), the EPC-known person scale was adapted to assess the amount of EPC with foreign individuals in particular $(\alpha=.91)$. Participants' ratings were averaged to form a synthetic score of EPC with

310 foreign individuals.

311 Attitudes toward foreign people. Participants were asked to "describe how you feel when

312 thinking about foreign people in general" by using six bipolar adjectives (e.g., warm/cold) on a

313 7-point scale ( $\alpha=.88$; Wright et al., 1997). Participants' ratings were averaged to form a general

314 score of intergroup prejudice. Higher values indicated a more positive attitude towards the 315 outgroup.

316 At last, participants reported their gender, age, nationality and native language. Participants

317 were then thanked and fully debriefed. This study was carried out in accordance with the Ethical 318 Committee of the University of Trieste (approval number 84) and in accordance with the 319 declaration of Helsinki.

\section{Results}

322 To verify whether higher amounts of EIPC predicted more positive intergroup attitudes we

323 performed a causal mediation analysis. The EIPC to intergroup attitudes relationship exhibited

324 both a direct and an indirect pathway through quality and/or quantity of contact with foreign

325 people. We extracted these pathways together with the indices of their statistical reliability, using

326 the mediation $\mathrm{R}$ software and performing a causal mediation based on linear mixed effect as

327 mediator model types. In particular path coefficients (i.e., $\beta \pm 1$ standard error of the mean) were 
328 estimated using linear mixed effect models fitted by minimizing the restricted maximum

329 likelihood criterion (Laird \& Ware, 1982). The advantages of this type of models over the more

330 traditional one based on mixed-model ANOVAs is discussed by Kliegl and colleagues (2010). In

331 particular, lme was shown to be more robust to unbalanced dataset and to suffer less severe loss

332 of statistical power compared to mixed-model ANOVAs. We followed Bates (2010) and used

333 this statistical procedure to obtain two-tailed p-values by means of likelihood ratio test based on

$334 \quad \chi^{2}$ statistics when contrasting lme with different complexities. Furthermore, we used type 3-like

335 two tailed p-values for significance estimates of lme's fixed effects and parameters adjusting for

336 the F-tests the denominator degrees-of freedom with the Satterthwaite approximation based on

337 SAS proc mixed theory. Finally, as indices of effect size, of the predictive power and of the

338 goodness of fit for the relevant paths estimated through lme models, we selected the Pearson- $r^{2}$

339 and the concordance correlation coefficient, the $r_{c}$. According to Vonesh, Chinchilli \& Pu, (1996;

340 but see also Rigutti, Fantoni \& Gerbino, 2015) this latter index provides an optimal measure of

341 the degree of agreement between the observed values and the lme predicted values, in the -1 to 1

342 range. As an additional measure of significant effect size associated to lme estimated coefficient,

343 we provided Cohen's $d$. To implement our mediation analysis, we used a default simulation type

344 quasi-Bayesian Monte Carlo method based on normal approximation (Imai, Keele, \& Tingley,

345 2010). In addition, a bootstrapping method with a number of re-samples large enough to

346 guarantee reliable results (i.e., 2000) was used to compute confidence intervals of the proportion

347 of effect mediated by quality and/or quantity of contact as inferred from the average causal

348 mediation (ACME), average direct (ADE) and average total effects. The main mediation model

349 we tested resulted from two preliminary analyses, contrasting competing models with increasing

350 complexities (i.e., degrees of freedom), but with the same random component. In particular, in 
351 both analyses participant gender and nationality were treated as both fixed and random intercepts

352 throughout our analysis for two major reasons: (1) We did not have any specific predictions on

353 the way gender and nationality might affect the EIPC to intergroup attitudes relationship; (2) We

354 aimed to maximize the robustness of the mediation analysis over individual variability.

355 In the first analysis a comparison of lme models with nested fixed effects showed that the

356 outcome variable was not affected by our balancing variable (i.e., order of presentation, $\chi^{2}{ }_{1}=$

$3570.02, p=.90)$. We thus excluded such a factor from the remaining analyses. The second

358 preliminary lme analysis was aimed at identifying the statistical structure of the lme model that

359 best represented the EIPC to intergroup attitudes relationship. The best representative structure

360 indeed might be characterized by either a multiplicative model including all interaction terms or

361 a simpler additive model including only the main effects. We selected the best model amongst

362 our two competing models thus entering the amount of EIPC as the predictor variable, quality

363 and quantity of intergroup contact as two independent mediators, and outgroup attitudes as the

364 outcome variable, with participant gender and nationality treated as both fixed and random

365 effects. Importantly, contrasting the two models, no interaction term turned out to be significant

$366\left(\chi^{2}{ }_{16}=22.43, p=.13\right)$ and we thus proceeded by performing the causal mediation analysis

367 treating our predictors as independent factors with gender and nationality not significantly

368 affecting the outcome variable $\left(F_{1,98}=0.30, p=.58\right.$ for nationality; $F_{1,98}=1.64, p=.20$ for

369 gender). This model accounted for a significant portion of the outcome variance $\left(r^{2}=0.34, r_{c}=\right.$

$370 \quad 0.51,95 \%$ CI $\left.[0.38-0.62], F_{5,98}=10.28, p<.001\right)$.

371

The model, shown in Figure 1, revealed a significant Total Effect, with higher amounts of

372 EIPC associated with more positive outgroup attitudes (consistent with $\mathrm{H} 1, \beta=0.41 \pm 0.12, d f=$

$373100, t=3.49, p<.001, d=0.70, r^{2}=0.18, r_{c}=0.304,95 \%$ CI [0.179- 0.419]). Importantly, the 
374 amount of EIPC contributed to the variance of outgroup attitudes, and also contributed to the 375 variance of both quality $\left(r^{2}=0.25, r_{c}=0.40,95 \%\right.$ CI $\left.[0.27-0.51], F_{1,100}=20.48, p<.001\right)$ and 376 quantity $\left(r^{2}=0.38, r_{c}=0.55,95 \% \mathrm{CI}[0.43-0.65], F_{1,100}=54.10, p<.001\right)$ of intergroup contact 377 (consistent with $\mathrm{H} 2)$. Also, quality $\left(r^{2}=0.33, r_{c}=0.50,95 \% \mathrm{CI}[0.37-0.61], F_{1,100}=37.28, p<\right.$ $378.001)$ and quantity $\left(r^{2}=0.14, r_{c}=0.24,95 \%\right.$ CI $\left.[0.12-0.35], F_{1,100}=6.75, p<.011\right)$ affected the 379 outcome variable. Furthermore, outgroup attitudes improved as quality $(\beta=0.59 \pm 0.10, d f=$ 380 $100, t=6.10, p<.001, d=1.22)$ and/or quantity $(\beta=0.27 \pm 0.10, d f=100, t=2.53, p<.01, d=$

381

382 383

384 385 386 387 388 389 390

0.51) of intergroup contact increased, which in turn enhanced with the increase of the amount of EIPC (quality: $\beta=0.45 \pm 0.10, d f=100, t=4.53, p<.001, d=0.91$; quantity: $\beta=0.69 \pm 0.09$, $d f=100, t=7.35, p<.001, d=1.47)$. Such a pattern of mutual relationships provided a strong ground for a potential mediation of the total effect by quality and/or and quantity of intergroup contact. The mediating role of quality of intergroup contact was attested by the fact that when it was added as a covariate to the effect of the amount of EIPC on outgroup attitudes, the direct association (i.e., Direct Effect) between the amount of EIPC and outgroup attitudes significantly decreased, reaching a coefficient statistically equal to zero $(\beta=0.17 \pm 0.12, d f=99, t=1.48, p=$ .14). Also, no significant loss in the fit was found when contrasting an lme model with quality as the only fixed effect vs. an lme model including all fixed effects (with $r_{c}$ slightly decreasing from $0.51,95 \%$ CI [0.38- 0.62] to $0.50,95 \%$ CI $\left.[0.37-0.60], \chi^{2}{ }_{1}=2.29, p=.13\right)$. By contrast, no such mediating effect was found for quantity of intergroup contact. No reliable modification of the direct association between the amount of EIPC and outgroup attitudes was found when quantity was included as a mediator $(\beta=0.35 \pm 0.15, d f=99, t=2.37, p=.02, d=0.48)$, with a significant loss in the fit when contrasting an lme model with quantity as the only fixed effect vs. an lme model including all fixed effects (with $r_{c}$ increasing from $0.24,95 \%$ CI [0.12 - 0.35] to 
$3970.31,95 \%$ CI [0.19- 0.43], $\left.\chi^{2}=5.74, p=.02\right)$. The Total Effect was accounted for by quality of

398 intergroup contact (consistent with $\mathrm{H} 3$ ), with a significant proportion of mediation $(0.58,95 \% \mathrm{CI}$

$399[0.27-1.21], p<.001)$ supported by the highly reliable Average Causal Mediation Effect

$400(\mathrm{ACME}=0.24,95 \% \mathrm{CI}[0.11-0.40], p<.001)$. No such mediation was found for quantity of

401 intergroup contact (proportion mediated $=0.15,95 \%$ CI $[-0.30-0.70], p=.49 ; \mathrm{ACME}=0.06$,

$40295 \%$ CI [-0.12 - 0.24], $p=.49)$.

403 To better ascertain the directionality of the relationship suggested by the previous

404 analysis, we proceeded by comparing the goodness-of-fit of the above specified mediation model

405 (i.e., Model 1) with that of an alternative mediation model, by inverting the directionality of the

406 relationship between the mediators and the predictor (i.e., Model 2). In this alternative model

407 only the Total Effect specified by the relation between quality of contact and outgroup attitudes

408 was significant $\left(\beta=0.56 \pm 0.10, d f=104, t=5.53, p<.001, d=1.08 ; r^{2}=0.33, r_{c}=0.50,95 \%\right.$

409 CI $\left.[0.37-0.61], F_{1,104}=30.6, p<.001\right)$. However, when the amount of EIPC was entered as a

410 mediator, this relationship still remained significant $(\beta=0.53 \pm 0.10, d f=98, t=4.89, p<.001$,

$411 d=0.99 ; r^{2}=0.34, r_{c}=0.51,95 \%$ CI $\left.[0.38-0.62], F_{1,98}=23.91, p<.001\right)$, thus proving that the

412 amount of EIPC did not mediate the relation between quality of contact and outgroup attitudes.

413 We further compared the two models. To attain this aim, we contrasted the two models

414 by using the Structural Equation Analyses (SEA), Lavaan R software (Rosseel, 2012). This

415 analyses allowed us to quantify the differential fit of the two models by means of distinct and

416 different indices, such as $\chi^{2} / d f$ ratio, SRMR, CFI and AIC. It is worth noting that, and according

417 to Hu and Bentler (1999), acceptable fit is revealed by a $\chi^{2} / d f$ ratio of less than 3, SRMR less

418 than .08 , and a CFI greater than or equal to .95 ; comparative measure of fit is also given by lower

419 levels of AIC in a model over the alternative model. Based on the analyses of these parameters, 
420 Model 1 showed a good fit of the data $\left(\chi^{2}=2.3, d f=1, p=.61\right.$; SRMR $=.07$; CFI $=.97$; $\mathrm{AIC}=$

421 954.8), and a higher goodness-of-fit than Model $2\left(\chi^{2}=15.52, d f=1, p=.05\right.$; SRMR $=.15, \mathrm{CFI}$

$422=.85 ; \mathrm{AIC}=975.7)$.

423

424 Discussion

425 This study aims at understanding the role played by intergroup physical contact in shaping

426 attitudes towards foreigners, and at testing the mediating role of intergroup contact (i.e., quantity 427 and quality) in this respect.

Results indicate that higher amounts of EIPC are associated with more positive outgroup 429 attitudes. This result confirms Seger and colleagues' (2014) findings, and suggests that the effect of physical contact goes beyond a brief and casual touch. Indeed, the EPC scale allowed us to assess individual differences in the amount of EIPC by capturing broader and detailed aspects of body-based encounters. Moreover, the amount of EIPC likely facilitates intergroup encounters, as testified by the fact that as the amount of EIPC increased, the frequency of intergroup interactions also increased. In addition, the amount of EIPC was also associated with the perceived quality of the intergroup contact. Indeed, higher amounts of EIPC were linked to more pleasant, less superficial and more cooperative intergroup interactions. Importantly, and in line with our hypothesis, only quality, and not quantity of intergroup contact, mediated the relation between the amount of EIPC and outgroup attitudes. However, one may claim that quantity, and quality of intergroup contact in particular, 440 would be linked to the amount of EIPC (and not vice versa), which in turn would mediate the 441 relation between intergroup contact and outgroup attitudes. Indeed, intergroup contact in general, 442 and quality of intergroup contact in particular, has been found to be predictive of social distance 
443 (Binder et al. 2009), which is also related to physical distance (Brewer, 1968). We directly tested

444 this alternative model (i.e., Model 2), which showed a lower goodness-of-fit than the

445 hypothesized one (i.e., Model 1). Moreover, the comparison between the two models showed

446 that quality of intergroup contact mediated the relation between the amount of EIPC and

447 outgroup attitudes, while the amount of EIPC did not mediate the relation between quality of

448 intergroup contact and outgroup attitudes. Hence, we may suggest that the amount of EIPC likely

449 plays a role in shaping the appraisal of intergroup encounters, since the reverse relation was not

450 supported by our data.

451

452 Conclusions

453 These results suggest that intergroup physical encounters may facilitate intergroup contact, and

454 therefore should be taken into account when discussing about strategies aimed at ameliorating

455 outgroup attitudes. Also, these results add further support to the existing relation between

456 intergroup physical contact and outgroup attitudes (i.e., Choma et al., 2014; Seger et al., 2014;

457 Hodson et al., 2015; Shamloo et al., in press), and for the first time shed light on the mediating

458 variables involved in this relation. We do believe these results may raise awareness on how

459 physical contact may represent a way to facilitate more pleasant relationships with the

460 individuals we communicate with, by enhancing the quality of these interactions and then

461 improving attitudes towards the group to which these individuals belong. This pattern of results

462 opens up to future studies which might experimentally vary the quantity of intergroup physical

463 contact (i.e., high frequency of physical contact vs. low frequency of physical contact), assess a

464 general appraisal of future intergroup contact, and test whether the variation in the frequency of 
465 intergroup physical contact shapes outgroup attitudes because it improves the anticipated quality

466 of the intergroup contact.

467 Nevertheless, some limits should be acknowledged. First, this is a correlational study and 468 we reckon this study to be exploratory. Although these results hint to the fact that intergroup 469 physical contact improves the quality of intergroup encounters, future studies should test this 470 hypothesis also by using a longitudinal approach and/or an experimental design, thus 471 ascertaining the causal direction of the variables in question.

472 Second, when considering participants' physical contact experience, their disposition 473 towards engaging in and receiving physical contact should be assessed (Webb \& Peck, 2015). 474 Also, given the cultural differences in terms of preferred interpersonal distances (Sorokowska et 475 al., 2017), willingness to engage in physical contact, and in the meaning associated with this type 476 of encounters (Remland, Jones, \& Brinkman, 1995), future studies should test the proposed 477 model in different cultural settings, thus strengthening its external validity. Despite the 478 significance of our findings more works are needed, especially experimental research is 479 requested to test the validity of the presented model. 


\section{Footnotes}

$482{ }^{1}$ Participants also rated the EPC scale referring to unknown people (i.e., EPC-stranger), albeit 483 this measure is beyond the scope of this pilot study. 
486

487

488

489

490

491

492

493

494

495

496

497

498

499

500

501

502

503

504

505

506

507

Allport GW. 1954. The nature of prejudice. Reading, MA: Addison-Wesley.

Asbrock F, Lemmer G, Becker JC, Koller J, \& Wagner U. 2014. "Who are these foreigners anyway?" The content of the term foreigner and its impact on prejudice. Sage Open, 4(2), 2158244014532819. https://doi.org/10.1177/2158244014532819

Bates D. 2010. lme4: mixed-effects modeling with R. New York, NY: Springer.

Bianchi M, Carnaghi A, Shamloo SE. 2018. Intergroup attitudes accessibility and motor approach-avoidance responses in White and Black individuals in Portugal. Psicologia Sociale, 2, 148-164

Binder J, Zagefka H, Brown R, Funke F, Kessler T, Mummendey A, ... \& Leyens J P. 2009. Does contact reduce prejudice or does prejudice reduce contact? A longitudinal test of the contact hypothesis among majority and minority groups in three European countries. Journal of Personality and Social Psychology, 96(4): 843-856. doi: $10.1037 / \mathrm{a} 0013470$

Blinder S, Allen, W. 2016. UK public opinion toward immigration: overall attitudes and level of concern. Available at: http://www.migrationobservatory.ox.ac.uk/resources/briefings/ukpublic-opinion-toward-immigration-overall-attitudes-and-level-of-concern/ (accessed 26 February 2018)

Brewer MB. 1968. Determinants of social distance among East African tribal groups. Journal of Personality and Social Psychology, 10(3): 279-289. doi:10.1037/h0026563

Brown R, \& Paterson J. 2016. Indirect contact and prejudice reduction: Limits and possibilities. Current Opinion in Psychology, 11: 20-24. doi:10.1016/j.copsyc.2016.03.005 
508 Choma BL, Charlesford JJ, \& Hodson, G. 2014. Reducing Prejudice with (Elaborated) Imagined 509 and Physical Intergroup Contact Interventions. Current Research in Social Psychology, 22(7), 20-26. https://uiowa.edu/crisp/sites/uiowa.edu.crisp/files/crisp22_7.pdf

511 Christ O, Hewstone M, Tausch N, Wagner U, Voci A, Hughes J, \& Cairns E. 2010. Direct 512 contact as a moderator of extended contact effects: Cross-sectional and longitudinal impact on outgroup attitudes, behavioral intentions, and attitude certainty. Personality and Social Psychology Bulletin, 36(12), 1662-1674.

516 Cohen J. 1988. Statistical power analysis for the behavioral sciences. Hillsdale, NJ: Erlbaum.

517 Crisp RJ, Husnu S, Meleady R, Stathi S, \& Turner, RN. 2010. From imagery to intention: A dual 518 route model of imagined contact effects. European Review of Social Psychology, 21(1), 188-236. https://doi.org/10.1080/10463283.2010.543312

Crisp RJ, Stathi S, Turner RN, \& Husnu S. 2009. Imagined intergroup contact: Theory, paradigm 521 and practice. Social and Personality Psychology Compass, 3(1), 1-18.

522 https://onlinelibrary.wiley.com/doi/abs/10.1111/j.1751-9004.2008.00155.x

523 Crisp RJ, \& Turner RN. 2009. Can imagined interactions produce positive perceptions?: Reducing prejudice through simulated social contact. American Psychologist, 64(4): 231. doi:10.1037/a0014718

Decety J, \& Grèzes J. 2006. The power of simulation: imagining one's own and other's behavior. Brain research, 1079(1), 4-14. https://doi.org/10.1016/j.brainres.2005.12.115

528 Duke MP, \& Nowicki S. 1972. A new measure and social-learning model for interpersonal 529 distance. Journal of Experimental Research in Personality, 6: 119-132.

530 Ehrlich HJ. 1973. The social psychology of prejudice. New York: Wiley 
531

532 Erceau D, \& Guéguen N. 2007. Tactile contact and evaluation of the toucher. The Journal of Social Psychology, 147(4): 441-444. doi:10.3200/SOCP.147.4.441-444

534 Fantoni C, Rigutti S, Piccoli V, Sommacal E, \& Carnaghi A. 2016. Faster but less careful 535 prehension in presence of high, rather than low, social status attendees. PloS one, 11(6), e0158095. https://doi.org/10.1371/journal.pone.0158095

537 Faul F, Erdfelder E, Lang AG, \& Buchner A. 2007. G* Power 3: A flexible statistical power analysis program for the social, behavioral, and biomedical sciences. Behavior research methods, 39(2), 175-191.

541

542

543

544

545

546

547

548

549

550

551

Field T. 2001. Touch. Cambridge, MA: MIT Press.

Fiske AP. 2004. Four modes of constituting relationships: Consubstantial assimilation; space, magnitude, time, and force; concrete procedures; abstract symbolism. In N. Haslam (Ed.), Relational models theory: A contemporary overview (pp. 61-146). Mahwah, NJ: Erlbaum.

Gaertner SL, Mann JA, Dovidio JF, Murrell AJ, \& Pomare M. 1990. How does cooperation reduce intergroup bias? Journal of Personality and Social Psychology, 59(4), 692-704. https://doi.org/10.1037/0022-3514.59.4.692

Gallace A, \& Spence C. 2010. The science of interpersonal touch: an overview. Neuroscience \& Biobehavioral Reviews, 34(2): 246-259. doi:10.1016/j.neubiorev.2008.10.004

Gallese V. 2001. The shared manifold hypothesis. From mirror neurons to empathy. Journal of consciousness studies, $8: 33-50$ 
552 Guéguen N, \& Fischer-Lokou J. 2003. Tactile contact and spontaneous help: An evaluation in a

553 natural setting. The Journal of Social Psychology, 143(6): 785-787. doi:

554 $10.1080 / 00224540309600431$

555 Gulledge AK, Gulledge MH, \& Stahmannn, RF. 2003. Romantic physical affection types and 556

557 relationship satisfaction. The American Journal of Family Therapy, 31(4): 233-242. doi:

558

559

560

561

562

563

564

565

566

567

568

569

570

571

572

573

$$
10.1080 / 01926180390201936
$$

Hertenstein MJ, Keltner D, App B, Bulleit BA, Jaskolka AR. 2006. Touch

communicates distinct emotions. Emotion 6: 528-533. doi: 10.1037/1528-3542.6.3.528

Hertenstein MJ, Verkamp JM, Kerestes AM, \& Holmes RM. 2006. The communicative functions of touch in humans, nonhuman primates, and rats: a review and synthesis of the empirical research. Genetic, Social, and General Psychology Monographs, 132(1): 5-94. doi:10.3200/MONO.132.1.5-94

Hodson G, Dube B, \& Choma BL. 2015. Can (elaborated) imagined contact interventions reduce prejudice among those higher in intergroup disgust sensitivity (ITG-DS)? Journal of Applied Social Psychology, 45(3), 123-131. https://doi.org/10.1111/jasp.12281

Holt-Lunstad J, Birmingham WA, \& LightKC. 2008. Influence of a "warm touch" support enhancement intervention among married couples on ambulatory blood pressure, oxytocin, alpha amylase, and cortisol. Psychosomatic medicine, 70(9): 976-985. doi:

\subsection{7/PSY.0b013e318187aef7}

Hu LT, \& Bentler PM. 1999. Cutoff criteria for fit indexes in covariance structure analysis: Conventional criteria versus new alternatives. Structural Equation Modeling: A Multidisciplinary Journal, 6(1): 1-55. doi:10.1080/10705519909540118 
574 Imai K, Keele L, \& Tingley D. 2010. A general approach to causal mediation

575 analysis. Psychological Methods, 15(4): 309-334. doi:10.1037/a0020761

576 Islam MR, \& Hewstone M. 1993. Dimensions of contact as predictors of intergroup anxiety,

577 perceived out-group variability, and out-group attitude: An integrative model. Personality

578 and Social Psychology Bulletin, 19(6): 700-710. doi:10.1177/0146167293196005

579 Istituto Nazionale di Statistica. 2018. Integrazione delle seconde generazioni: microdati ad uso 580 pubblico [The integration of the second generation: microdata for public use]. Retrieved

581 from: https://www.istat.it/it/archivio/210741

582 Jones SE, Yarbrough AE, 1985. A naturalistic study of the meaning of touch.

583 Communication Monographs, 52: 19-56. doi: 10.1080/03637758509376094

584 Kawakami K, Phills CE, Steele JR, \& Dovidio JF. 2007. (Close) distance makes the heart grow 585 fonder: Improving implicit racial attitudes and interracial interactions through approach

586

587 behaviors. Journal of personality and social psychology, 92(6), 957. http://dx.doi.org/10.1037/0022-3514.92.6.957

Kliegl R, Masson ME, \& Richter EM. 2010. A linear mixed model analysis of masked repetition 589 priming. Visual Cognition, 18(5), 655-681. https://doi.org/10.1080/13506280902986058

Kosslyn SM, Ganis G, \& Thompson WL. 2001. Neural foundations of imagery. Nature Reviews: Neuroscience, 2(9), 635-642. doi: 10.1038/35090055

Kraus, MW, Huang C, \& Keltner D. 2010. Tactile communication, cooperation, and performance: an ethological study of the NBA. Emotion, 10(5): 745. doi:

594 10.1037/a0019382. 963-974. doi: $10.2307 / 2529876$ 
597 Lemmer G, \& Wagner U. 2015. Can we really reduce ethnic prejudice outside the lab? A

598 meta-analysis of direct and indirect contact interventions. European Journal of Social

599 Psychology, 45(2): 152-168. doi: 10.1002/ejsp.2079

600 Liebkind K, \& McAlister AL. 1999. Extended contact through peer modelling to promote

601

602 tolerance in Finland. European Journal of Social Psychology, 29(5-6), 765-780.

603 https://doi.org/10.1002/(SICI)1099-0992(199908/09)29:5/6<765::AIDEJSP958>3.0.CO;2-J

Mazziotta A, Mummendey A, \& Wright SC. 2011. Vicarious intergroup contact effects: Applying social-cognitive theory to intergroup contact research. Group Processes \& 607 608 609 610 Intergroup Relations, 14(2): 255-274. doi:10.1177/1368430210390533

Paladino MP, \& Castelli L. 2008. On the Immediate Consequences of Intergroup Categorization: Activation of Approach and Avoidance Motor Behavior Toward Ingroup and Outgroup Members. Personality and Social Psychology Bulletin, 34(6), 755-768. https://doi.org/10.1177/0146167208315155 effects on prejudice: A normative interpretation. International Journal of intercultural relations, 31(4), 411-425. https://doi.org/10.1016/j.ijintrel.2006.11.003

Pettigrew TF, \& Tropp LR. 2006. A meta-analytic test of intergroup contact theory. Journal of Personality and Social Psychology, 90(5): 751. doi: 10.1037/0022-3514.90.5.751 public views towards foreigners in Germany and Israel. European Sociological Review, 19(4), 379-392. https://doi.org/10.1093/esr/19.4.379 
619 Rankin RE, \& Campbell DT. 1955. Galvanic skin response to Negro and white experimenters. 620 The Journal of Abnormal and Social Psychology, 51(1): 30-33. doi:10.1037/h0041539

621 Remland MS, Jones TS, \& Brinkman H. 1995. Interpersonal distance, body orientation, and 622 touch: Effects of culture, gender, and age. The Journal of Social Psychology, 135(3): 281-

623 297. doi: $10.1080 / 00224545.1995 .9713958$

624 625 environment. PeerJ, 3:e828. doi: 10.7717/peerj.828

626

627 Software, 48(2): 1-36. doi:10.18637/jss.v048.i02

628

629

630

631

632

633

634

635

636

637

638

639

640

641

Rigutti S, Fantoni C, \& Gerbino W. 2015. Web party effect: a cocktail party effect in the web

Rosseel Y. 2012. lavaan: An R Package for Structural Equation Modeling. Journal of Statistical

Seger CR, Smith ER, Percy EJ, \& Conrey FR. 2014. Reach Out and Reduce Prejudice: The Impact of Interpersonal Touch on Intergroup Liking. Basic and Applied Social Psychology, 36(1): 51-58. doi:10.1080/01973533.2013.856786

Shamloo SE, Carnaghi A, Picccoli V, Grassi M, Bianchi M. In press. Imagined Intergroup Physical Contact Improves Attitudes Towards Immigrants. Frontiers in Psychology

Smith ER. 2008. Social relationships and groups: New insights on embodied and distributed cognition. Cognitive Systems Research, 9(1-2), 24-32. https://doi.org/10.1016/j.cogsys.2007.06.011

Sorokowska A, Sorokowski P, Hilpert P, Cantarero K, Frackowiak T, Ahmadi K, ... \& Blumen S. 2017. Preferred Interpersonal Distances: A Global Comparison. Journal of CrossCultural Psychology, 48(4): 577-592. doi:10.1177/0022022117698039

Stephan WG, Diaz-Loving R, \& Duran A. 2000. Integrated threat theory and intercultural attitudes. Journal of Cross-Cultural Psychology, 31: 240-249. doi:10.1177/0022022100031002006 
642 Turner RN, Crisp RJ, \& Lambert E. 2007. Imagining Intergroup Contact Can Improve 643 Intergroup Attitudes. Group Processes \& Intergroup Relations, 10(4), 427-441.

644 https://doi.org/10.1177/1368430207081533

645 Turner RN, Hewstone M, \& Voci A. 2007. Reducing explicit and implicit outgroup prejudice via 646 direct and extended contact: The mediating role of self-disclosure and intergroup anxiety. Journal of personality and social psychology, 93(3), 369.

648 http://dx.doi.org/10.1037/0022-3514.93.3.369

649

650

651

652

653

654

655

656

657

658

659

660

661

662

663 police: The roles of quality of contact, attitudes towards the behaviour and subjective norms. British Journal of Social Psychology, 45(2): 285-302. doi:

\section{$10.1348 / 014466605 X 49618$}

Voci A, \& Hewstone M. 2003. Intergroup contact and prejudice toward immigrants in Italy: The mediational role of anxiety and the moderational role of group salience. Group Processes \& Intergroup Relations, 6(1): 37-54. doi: 10.1177/1368430203006001011

Vonesh EF, Chinchilli VM, \& Pu K. 1996. Goodness-of-fit in generalized nonlinear mixedeffects models. Biometrics, 572-587. doi: 10.2307/2532896

Vrana SR, \& Rollock D. 1998. Physiological response to a minimal social encounter: Effects of gender, ethnicity, and social context. Psychophysiology, 35(4): 462-469. doi:

\section{$10.1111 / 1469-8986.3540462$}

Webb A, \& Peck J. 2015. Individual differences in interpersonal touch: On the development, validation, and use of the "comfort with interpersonal touch" (CIT) scale. Journal of Consumer Psychology, 25(1): 60-77. doi: 10.1016/j.jcps.2014.07.002 
664 Wiltermuth SS, \& Heath C. 2009. Synchrony and cooperation. Psychological science, 20(1), 1-5. 665 https://doi.org/10.1111/j.1467-9280.2008.02253.x

666 Wright SC, Aron A, McLaughlin-Volpe T, \& Ropp SA. 1997. The extended contact effect:

667 Knowledge of cross-group friendships and prejudice. Journal of Personality and Social $668 \quad$ psychology, 73(1): 73-90. doi:10.1037/0022-3514.73.1.73 
671 How many times have you held the hand of a $\mathbf{X}^{1}$ ?

672 How many times have you walked arm in arm with a $\mathbf{X}$ ?

673 How many times have you caressed a $\mathbf{X}$ ?

674 How many times have you got a massage from a $\mathbf{X}$ ?

675 How many times have you got a hair wash by a $\mathbf{X}$ ?

676 How many times have you put your hand on the shoulder of a $\mathbf{X}$ ?

677 How many times have you sat on the knees of a $\mathrm{X}$ ?

678 How many times have you hugged a $\mathbf{X}$ ?

679 How many times have you shaken hands with a $\mathbf{X}$ ?

680 How many times have you kissed a $\mathbf{X}$ ?

681 How many times have you stood shoulder to shoulder with a $\mathbf{X}$ ?

682 How many times have you high-fived a $\mathbf{X}$ ?

683

${ }^{1}$ In the EPC scale the "X" was replaced with different target groups for the pilot $(\mathrm{X}=$ known person) and for the main study ( $\mathrm{X}=$ foreign person). 


\section{Table $\mathbf{1}$ (on next page)}

Factor loadings of the Principle Component Analysis on the EPC-known person items in the Pilot Study.

Note. EPC-known person = experienced physical contact with a known person; EPC-known person from 1 to 12 refer to the twelve items used in the scale. 


\begin{tabular}{lc}
\hline & Component \\
\cline { 2 - 2 } Items & 1 \\
\hline EPC-known person 1 & .75 \\
EPC-known person 2 & .80 \\
EPC-known person 3 & .83 \\
EPC-known person 4 & .68 \\
EPC-known person 5 & .45 \\
EPC-known person 6 & .72 \\
EPC-known person 7 & .67 \\
EPC-known person 8 & .79 \\
EPC-known person 9 & .51 \\
EPC-known person 10 & .73 \\
EPC-known person 11 & .62 \\
EPC-known person 12 & .65 \\
\hline
\end{tabular}

1 Note. EPC-known person = experienced physical contact with a known person; EPC-known 2 person from 1 to 12 refer to the twelve items used in the scale. 


\section{Figure 1}

Causal Ime mediation analysis on attitudes towards foreigners with the amount of experienced intergroup physical contact (EIPC) as predictor variable and quality and quantity of intergroup contact as mediators (assessed independently).

Coefficients marked with one, two or three asterisks are significant at $p<.05, p<.01$ and $p$ $<.001$ level, respectively. The effects of the predictor variable on the mediators (i.e., quality and quantity of intergroup contact) are shown on the arrow lines connecting EIPC to the mediators. The contribution of the mediators to outgroup attitudes is depicted on the line connecting the mediators to outgroup attitudes. The Ime estimates of the Total Effect of the predictor variable on attitudes is included in the rightmost part of the model next to the Attitudes box. The Direct effects (with quality and quantity as mediators) are depicted in the middle part of the model. The proportion of effect mediated by quality and quantity of intergroup contact is depicted above the line connecting the mediators to attitudes, following the arrows. 


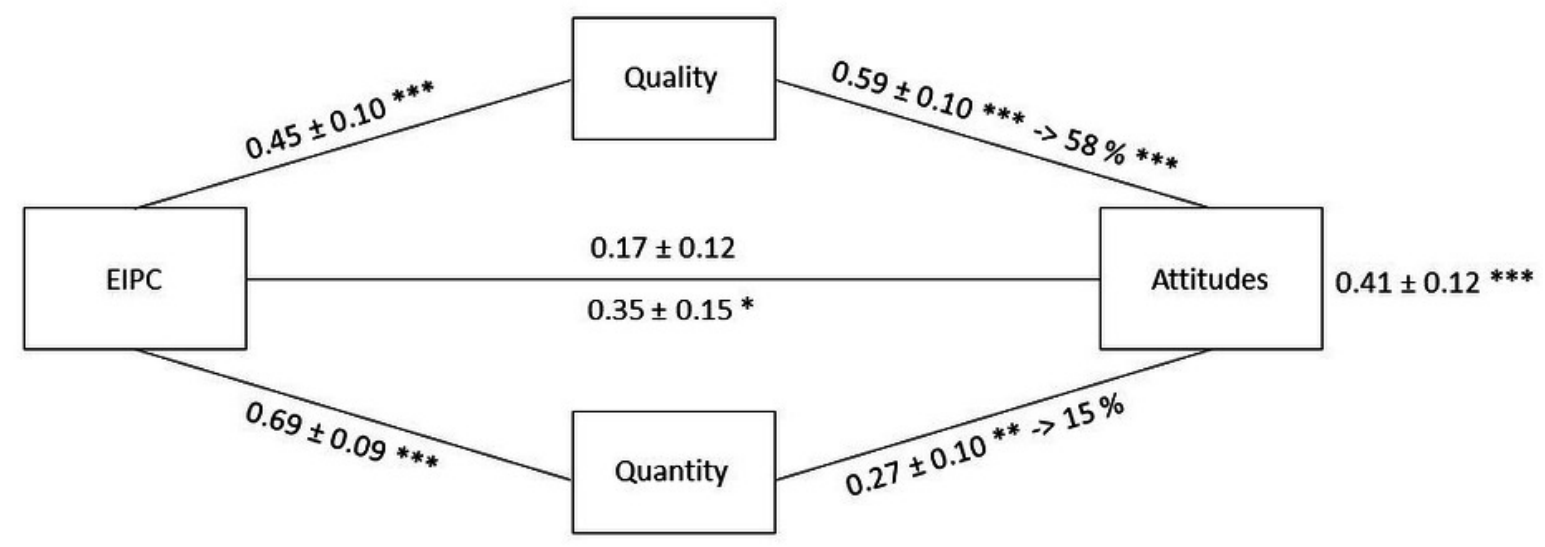

